\title{
Connectedness affects dot numerosity judgment: Implications for configural processing
}

\author{
LIXIA HE \\ State Key Laboratory of Brain and Cognitive Science, Institute of Biophysics, \\ Chinese Academy of Sciences, Beijing, China \\ JUN ZHANG \\ University of Michigan, Ann Arbor, Michigan \\ AND \\ Tiangang Zhou And Lin Chen \\ State Key Laboratory of Brain and Cognitive Science, Institute of Biophysics, \\ Chinese Academy of Sciences, Beijing, China
}

\begin{abstract}
Participants judged the number of dots in visual displays with brief presentations $(200 \mathrm{msec})$, such that the numerosity judgment was based on an instantaneous impression without counting. In some displays, pairs of adjacent dots were connected by line segments, whereas, in others, line segments were freely hanging without touching the dots. In Experiments 1, 2A, and 2B, connecting pairs of dots by line segments led to underestimation of dot numbers in those patterns. In Experiment 3, we controlled for the number of freely hanging line segments, whereas Experiment 4 showed that line segments that were merely attached to dots without actually connecting them did not produce a considerable underestimation effect. Experiment 5 showed that a connectedness effect existed when stimulus duration was reduced $(50 \mathrm{msec})$ or extended $(1,000 \mathrm{msec})$. We conclude that connectivity affects dot numerosity judgments, consistent with earlier findings of a configural effect in numerosity processing. Implications of the role of connectedness in object representation are discussed.
\end{abstract}

The term numerosity judgment refers to the estimation of the number of dots in a pattern made of a multitude of dots, or to comparing the dot numbers of two such patterns, under a short presentation duration to preclude overt or covert counting, with the judgment based solely on an instantaneous impression of numerosity. In a typical numerosity comparison task, a participant is briefly presented with two visual displays of dots and is asked to indicate which contains more dots. In a typical numerosity estimation task, a participant is asked to provide an estimate of the dot number in a display without actually performing one-by-one counting. Earlier researchers (Indow \& Ida, 1977; Krueger, 1972, 1984) constructed psychophysical scales and described numerosity as a power function of the number of items in the stimulus (with an exponent of around 0.85). A participant's ability to discriminate numerosity was found to be invariant against (i.e., irrespective of) dot size (Allik, Tuulmets, $\&$ Vos, 1991), although his or her ability to estimate numerosity bore an inverse relationship to dot size (Ginsburg $\&$ Nicholls, 1988). Errors in discrimination of numerosity were shown to be related not only to the randomness (external variance) of dots-per-unit area in the dot patterns themselves but also to the internal noise (observer variance) generated by the observer (Burgess \& Barlow, 1983).
A well-documented finding in numerosity research is that spatial configuration of elements (dots) exerts a profound influence on perceived numerosity: Compared with dots in random distribution, dots in regular arrangements (such as in concentric circles) tend to be overestimated, whereas dots forming clusters are underestimated (Allik \& Tuulmets, 1991; Ginsburg, 1978; Ginsburg \& Goldstein, 1987). The same pattern of results was replicated in children 5.8-14.6 years of age (Ginsburg \& Deluco, 1979). These findings were explained by the so-called "occupancy model" (Allik \& Tuulmets, 1991), which claimed that perceived numerosity depended on the subjective area covered by the totality of dots in a dot pattern; the impact of each dot was postulated to have some local spatial spread into its immediate neighborhood. Each dot supposedly occupied a circular territory of a fixed radius larger than its physical size, so when two dots were close to one another, there was an overlap in their individual territory such that the total effective region covered by the dots was smaller than it would have been if the two dots had been more widely separated. Decreasing the distance of adjacent dots - according to the occupancy modelcaused more overlapping apparent area, leading to underestimation of dot numerosity.

J.Zhang, junz@umich.edu 
In addition to the effect of the spatial arrangement of dots on perceived numerosity, there are nonspatial, structural factors affecting numerosity perception. One such nonspatial factor is dot connectedness. In a preliminary study, Koesling, Carbone, Pomplun, Sichelschmidt, and Ritter (2004) reported that, when dots were connected in order to form the shape of a closed polygon, they appeared much less numerous than did those in an unconnected dot display. Comparing their Experiment 2 with their Experiment 1 suggests both that merely connecting the dots to form polygons will result in underestimation and that forming an enclosed figure is not a necessary condition for this illusion. However, Koesling et al. (2004) used a method of adjustment whereby participants adjusted the number of dots in a comparison stimulus to match that in a target display, without restriction to their viewing time. Although the dots in the visual displays were too numerous for one-by-one counting, an eyetracker record revealed that participants nevertheless performed patch-by-patch comparisons (Koesling, Pomplun, \& Ritter, 1998) instead of forming an instantaneous impression of dot numerosity, as was done in previous paradigms.

In the present study, we investigated how simply connecting the dots by line segments affected numerosity perception in briefly presented visual displays. We employed a forced choice design with varying numbers of dots (9-15) in each display and obtained psychometric functions for numerosity discrimination. In Experiment 1, we manipulated the number of connecting lines in test patterns while controlling for the total number of lines. We showed that, when adjacent dots were connected, an underestimation of numerosity occurred in a manner that directly depended on the number of connected dot pairs. In Experiment 2, we validated this finding by applying connectedness to reference patterns themselves. In Experiment 3, we controlled for the number of freely hanging lines to rule out one explanation - that dot number underestimation is due to a more orderly and less dense appearance in those patterns with connected dots. In Experiment 4, using dot patterns in which only one end of a line segment was allowed to connect to a dot, we checked for the possibility that dots at the two ends of a line segment are unintentionally suppressed during numerosity processing. Experiment 5 showed a consistent connectedness effect when the presentation duration was reduced to $50 \mathrm{msec}$ or prolonged to $1,000 \mathrm{msec}$. Such a configural effect can be attributed only to perceptual binding (grouping) of dots: Dots connected by a line segment form unitary objects. The implications for object representation will be discussed.

\section{EXPERIMENT 1}

\section{Method}

Participants. Four university students participated in the experiment and received hourly pay. They were all naive as to the purpose of the experiment.

Stimuli. Stimuli were presented on a 22 -in. computer monitor (ViewSonic P220f) with a resolution of $1,024 \times 768$ pixels $(1$ pixel $=$ $0.0275^{\circ}$ at a viewing distance of $80 \mathrm{~cm}$, where participants were seated). Two visual patterns generated by the MATLAB program, one serving as a reference and the other as a test, were displayed simultaneously in a participant's left and right hemifields. Each pattern had a size of $160 \times 240$ pixels, was grayscale $($ RGB indices in MATLAB were $102,102,102$ ), and was presented against a black background (RGB indices were set to $0,0,0)$. A total of 168 reference patterns were first created off-line, each with 12 randomly distributed black dots of circular shape with a diameter of 12 pixels and four black line segments of 2-pixel width and of varying length (30-60 pixels). Dots were separated by a minimum distance of 42 pixels (measured from their centers) and were a minimum of 10 pixels from the four boundaries of the pattern but were otherwise randomly distributed within each pattern. Lines were generated with random orientations and spatial positions, subject to the constraints that (1) they did not cross each other, (2) they did not extend beyond the pattern boundaries, and (3) the distance from any point on the line to the center of any dot was greater than 12 pixels, with the exception of dot-connecting lines. Then, a total of 168 different test patterns were created off-line. In the test patterns, an individual line could link two adjacent dots (i.e., the endpoints of the line were each at the center of a dot) to form a connected object or could otherwise hang freely (as in the reference patterns). There were three levels of connectedness, depending on how many pairs of dots were connected by lines, respectively called the zero-connected, one-connected, and two-connected patterns (see examples in Figure 1). The number of dots in the test patterns varied symmetrically around the reference number 12 across seven levels: $9,10,11,12,13,14$, and 15 . The 168 test patterns were generated in MATLAB through the following procedure. First, for each of the 3 (connectedness) $\times 7$ (number of dots) $=21$ test conditions, 8 different patterns were created for the zero-connected condition, giving rise to a total of 56 patterns with different spatial configurations of dots. Note that they were essentially the same (apart from the numbers of dots) as the reference patterns, in which no dots
A

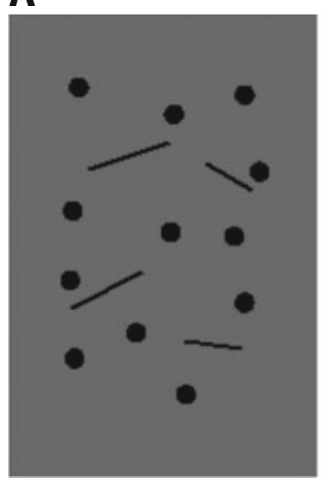

B

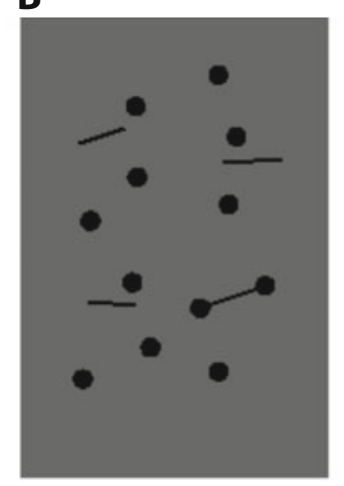

C

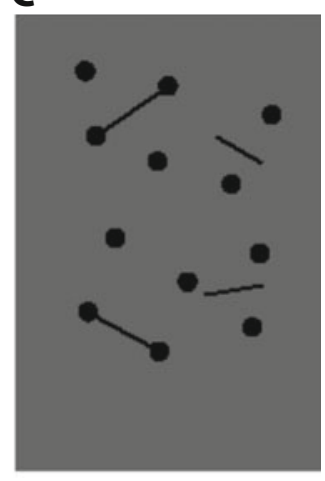

Figure 1. Examples of test patterns at different levels of dot connectedness - zero connected (A), one connected (B), and two-connected (C)—in Experiments 1, 2A, and $2 B$. 
were connected by lines. After the zero-connected patterns were generated, 56 one-connected test patterns were generated by modifying each zero-connected pattern in such a way that one of the lines was randomly selected, erased, and replaced by another line drawn to connect two randomly selected dots with a center-to-center dot spacing ranging from 30 to 60 pixels, then the whole figure was flipped either horizontally or vertically. (Note that the mirror-imaged figures were all used as test patterns and were not paired with the originals as reference patterns.) Another 56 two-connected test patterns were generated from the one-connected patterns in a similar fashion by connecting another pair of dots as described above. All patterns (reference or test) had a total of four lines in this experiment, either freely hanging or connecting pairs of dots.

Design. During each trial, a reference and a test pattern were simultaneously displayed on the two hemifields for $200 \mathrm{msec}$, followed by a blank screen. After the participant's response (a manual keypress), a new trial started, following a random delay of 500 $1,000 \mathrm{msec}$. No feedback was given, and there was no requirement on response speed. We randomized presentation of reference and test patterns in the left and right sides of the fixation cross. An experimental session consisted of five blocks, with each block consisting of 336 trials. In the first half of each block (168 trials), each of the 21 conditions was repeated eight times; the conditions and the location of test/reference patterns were completely randomized. In the second half of each block (168 trials), the presentation order for the various conditions replicated that of the first half, whereas the locations of the test/reference patterns were reversed so that the stimulus displays were totally balanced. Across the five blocks, there was a total of 80 trials in each condition (contributing to one data point on the psychometric function; see below). Participants were given a brief recess between blocks.

Procedure. The participants were seated about $80 \mathrm{~cm}$ from the front of the computer monitor in a room with dim ambient light and were instructed to maintain fixation at the green cross at the center of the screen. The task was to determine whether the left or right pattern had more dots by pressing one of the labeled keys. Before each experiment, 30 practice trials were given with relatively easier tasks (i.e., discriminating a 9-dot pattern from a 12-dot pattern). The participants were allowed to ask additional questions before starting the experiment, which lasted roughly $1 \mathrm{~h}$ (excluding instruction and practice).

\section{Results}

Participants' performances for each of the 21 conditions were quantified as the percentage of the test patterns judged to contain more dots than the 12-dot reference patterns among a total of 80 trials of any single condition. Psychometric functions for the zero-, one-, and two-connected conditions were generated by fitting a cumulative Gaussian sigmoid curve using the Psignifit toolbox software for MATLAB (v. 2.5.6; see bootstrap-software.org/psignifit/), which implements the maximum-likelihood method described by Wichmann and Hill (2001a). In fitting the psychometric curves, both gamma and lambda parameters, which, respectively, control the low and high asymptote/plateau of the curve, were allowed to vary in the default range of $0-0.05$, taking into account the occasional but unavoidable lapse in performance. Figure 2 plots the data of the 4 participants under different connectedness conditions, along with the bestfitting curves as provided by the Psignifit software under the cumulative Gaussian model. The abscissa represents the number of dots in the test patterns, whereas the ordinate represents the proportion/percentage of such test patterns (of a particular dot-number and connectedness condition) judged to contain more dots than the 12-dot reference patterns across 80 such trials. The $50 \%$ threshold value, plus or minus $(\gamma+\lambda) / 2$, represents chance performance, and the corresponding number of dots, the point of subjective equality (PSE) where dot numerosity of the test patterns was judged to subjectively equal the numerosity of the 12-dot reference patterns (where no dots were connected by lines). For all participants, their psychometric curves for the three connectedness conditions shift systematically. Take the representative data of Participant H.W.U. as an example: The PSEs for the zero-, one-, and two-connected conditions are 11.89, 12.60, and 13.49 , respectively. The data from all 4 participants in Figure 2 demonstrate that consistent rightward shifts in the psychometric functions occurred as the number of connected pairs of dots increased.

To ensure that the underestimation of numerosity for one- and two-connected patterns is independent of the form of psychometric function (cumulative Gaussian) chosen to fit the data, we used other sigmoidal forms, such as the logistic and Weibull functions to perform curve fitting as well. The best-fitting PSE values, the slope values, and the lapses (lambda and gamma) for all three fitting functions are given in Tables 1A-1D, respectively. There is virtually no difference between fittings by a cumulative Gaussian function and those by a logistic function. The systematic dependence of PSE on the level of connectedness is robust across all three fitting functions.

\section{EXPERIMENTS 2A AND 2B}

\section{Method}

Participants. Two university students participated in Experiment 2A, and 2 participated in Experiment 2B. All participants were naive as to the purpose of the experiments. None of them had participated in Experiment 1.

Stimuli. The stimuli were similar to those used in Experiment 1, except that, here, the reference patterns were 12-dot figures with one pair of dots connected by a line (one connected, Experiment 2A) or with two pairs of dots connected by two lines (two connected, Experiment 2B).

\section{Results}

The same analysis (as that described for Experiment 1) was performed on data collected in these experiments. Psychometric functions were fitted using the cumulative Gaussian function (see Figure 3). For the 2 participants in Experiment 2A, where the reference patterns were all 12-dot one-connected patterns, the PSEs for the zero-, one-, and two-connected test patterns were 11.26, 11.75, and 12.44 for Participant Y.F.Z., and 11.51, 12.02, and 12.56 for Participant R.H.E. There was a consistent leftward shift of all three psychometric functions obtained from Experiment 2A relative to those obtained from Experiment 1 , indicating that the perceived numerosity of dots in a reference pattern has also been affected by the presence/absence of connecting lines. For the 2 participants in Experiment 2B, where the reference patterns were all 12-dot two-connected patterns, the PSEs were 10.74, 11.30, and 12.00 for Participant S.Y.L. and 10.70, 11.29, and 11.99 for Participant Y.F.L. for the zero-, one-, and two-connected test patterns, respectively. All three psy- 
A
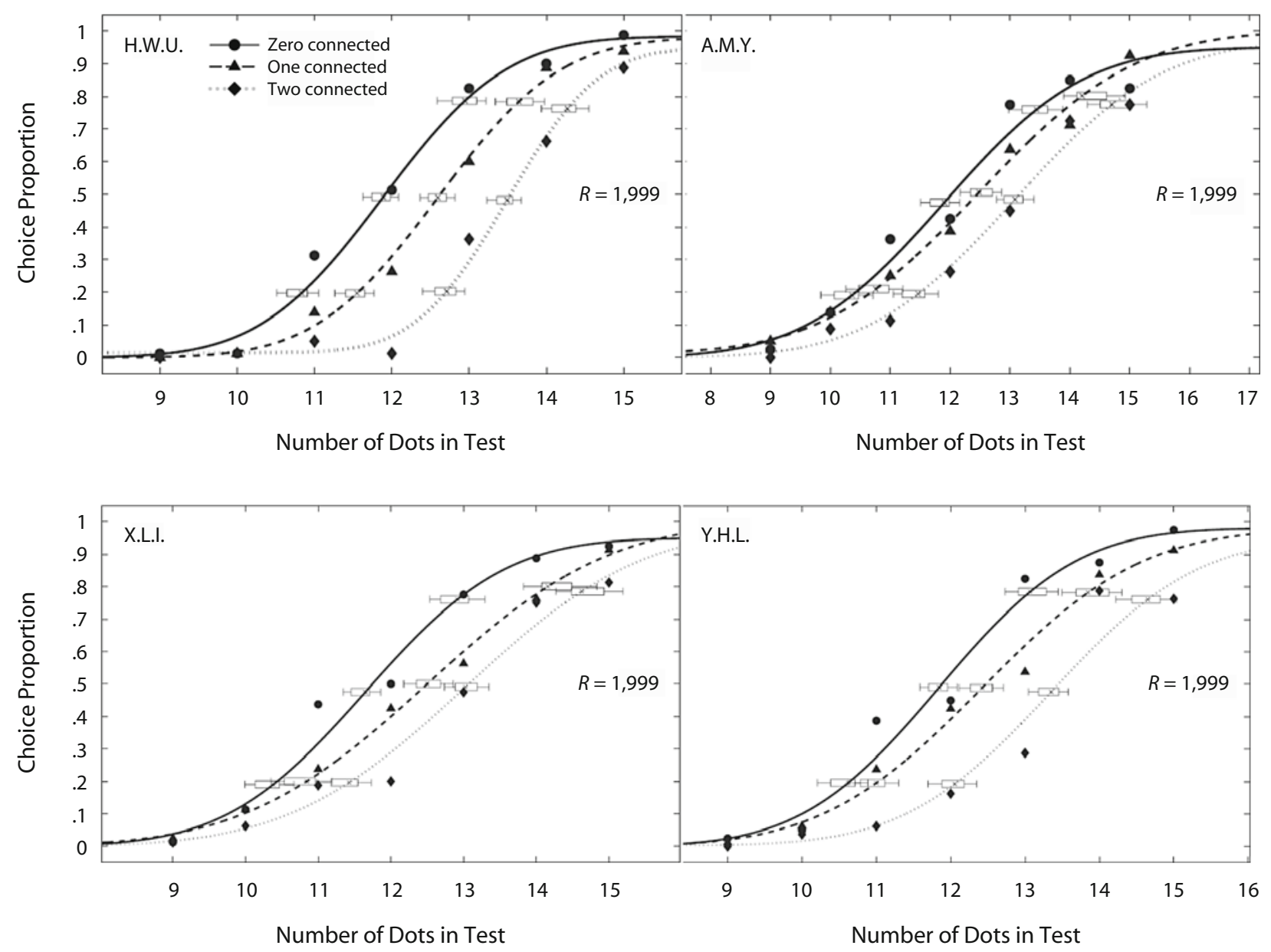

B

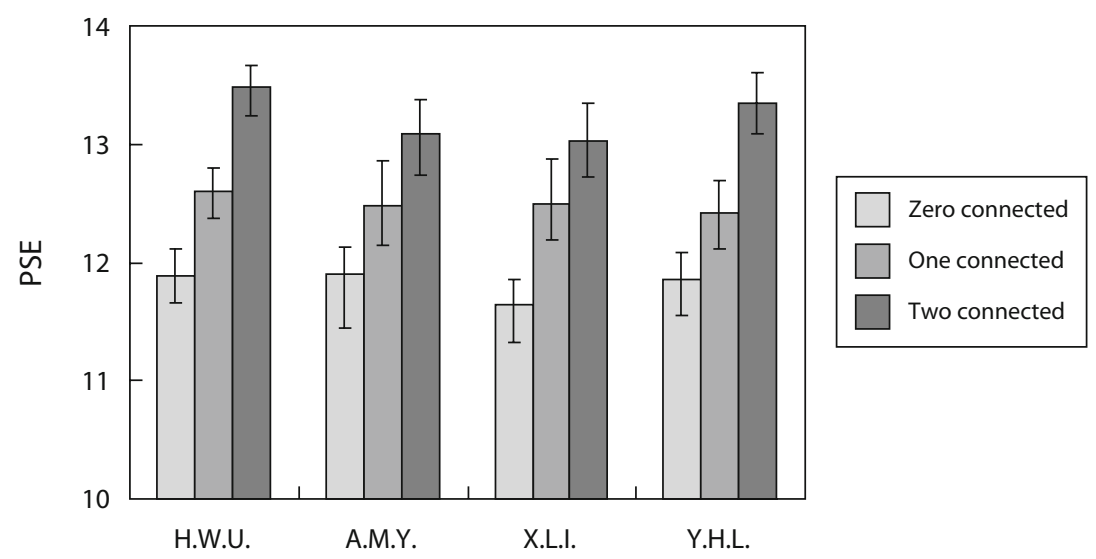

Figure 2. Results of Experiment 1. (A) Psychometric functions of the 4 participants. The proportion of trials that the test patterns were judged to contain more dots than the 12-dot reference pattern is plotted against the actual number of dots in the test patterns. Error bars around the $.2, .5$, and .8 proportions indicate $95 \%$ confidence intervals of numerosity estimation, which were found with the Psignifit toolbox using the bootstrap method (see Wichmann \& Hill, 2001b). Here $R$ refers to the number $(1,999)$ of bootstrap runs used in fitting and simulating. (B) Plot of points of subjective equality (PSEs) for the 4 participants in the different connectedness conditions. 
Table 1A

Point of Subjective Equality Values Using Cumulative Gaussian, Logistic, and Weibull Functions

\begin{tabular}{cccc}
\hline & \multicolumn{3}{c}{ Curve-Fitting Function } \\
\cline { 2 - 3 } Condition & Gaussian & Logistic & Weibull \\
\hline Zero connected & 11.89 & 11.90 & 11.97 \\
One connected & 12.60 & 12.75 & 12.79 \\
Two connected & 13.49 & 13.53 & 13.65 \\
& Participant & A.M.Y. \\
Zero connected & 11.90 & 11.88 & 12.01 \\
One connected & 12.48 & 12.48 & 12.51 \\
Two connected & 13.08 & 13.21 & 13.16 \\
& Participant X.L.I. & \\
Zero connected & 11.65 & 11.64 & 11.74 \\
One connected & 12.50 & 12.51 & 12.53 \\
Two connected & 13.03 & 12.98 & 13.05 \\
& Participant Y.H.L. & \\
Zero connected & 11.85 & 11.87 & 11.88 \\
One connected & 12.42 & 12.45 & 12.47 \\
Two connected & 13.35 & 13.14 & 13.55 \\
\hline
\end{tabular}

Table 1C

Lambda Values Using Cumulative

Gaussian, Logistic, and Weibull Functions

\begin{tabular}{cccc}
\hline \multirow{2}{*}{ Condition } & \multicolumn{3}{c}{ Curve-Fitting Function } \\
\cline { 2 - 4 } Gaussian & \multicolumn{1}{c}{ Logistic } & Weibull \\
\hline Zero connected & 0.0162599 & 0.000510539 & 0.025087 \\
One connected & 0.0195026 & 0.0195558 & 0.0499955 \\
Two connected & 0.0499929 & 0.0499948 & 0.0499968 \\
& \multicolumn{4}{c}{ Participant A.M.Y. } \\
Zero connected & 0.0499983 & 0.0499975 & 0.0499999 \\
One connected & $5.42187 \cdot 10^{-6}$ & $6.25786 \cdot 10^{-6}$ & 0.0143164 \\
Two connected & 0.030405 & 0.0187585 & 0.0499943 \\
& \multicolumn{4}{c}{ Participant X.L.I. } & \\
Zero connected & 0.0499961 & 0.0499935 & 0.0499881 \\
One connected & $1.87519 \cdot 10^{-5}$ & $4.12792 \cdot 10^{-5}$ & 0.032551 \\
Two connected & 0.0190147 & 0.0398539 & 0.0499919 \\
& \multicolumn{2}{c}{ Participant Y.H.L. } & \\
Zero connected & 0.0193312 & 0.0108014 & 0.0478665 \\
One connected & 0.0223532 & 0.0168233 & 0.0499919 \\
Two connected & 0.0499485 & 0.0499991 & 0.0205951 \\
\hline
\end{tabular}

chometric curves consistently were shifted to the left relative to those in Experiment 2A and, of course, to those in Experiment 1, indicating that a participant's perceived numerosity of dots in the reference pattern is affected to a greater degree. Taken together, Experiment 1 and Experiments $2 \mathrm{~A}$ and $2 \mathrm{~B}$ show that connecting pairs of dots in these displays tends to decrease perceived numerosity in a manner that is directly related to the number of pairs of dots connected by lines.

\section{EXPERIMENT 3}

\section{Method}

Participants. Three university students participated, and they were all naive as to the purpose of the experiment. None had participated in any of the previous experiments.
Table 1B

Slope Values at the Point of Subjective Equality Using Cumulative Gaussian, Logistic, and Weibull Functions

\begin{tabular}{cccc}
\hline & \multicolumn{3}{c}{ Curve-Fitting Function } \\
\cline { 2 - 3 } Condition & Gaussian & Logistic & Weibull \\
\hline Zero connected & .3177 & .3196 & .3032 \\
One connected & .3206 & .3396 & .3504 \\
Two connected & .4292 & .4061 & .3688 \\
& Participant & A.M.Y. \\
Zero connected & .2179 & .2329 & .2119 \\
One connected & .1948 & .2019 & .1972 \\
Two connected & .2075 & .2220 & .2227 \\
& Participant X.L.I. & \\
Zero connected & .2651 & .2822 & .2676 \\
One connected & .2025 & .2147 & .2144 \\
Two connected & .2099 & .2306 & .2297 \\
& Participant Y.H.L. & \\
Zero connected & .2745 & .2900 & .2904 \\
One connected & .2359 & .2497 & .2508 \\
Two connected & .2588 & .2888 & .2661 \\
\hline
\end{tabular}

\section{Table 1D}

Gamma Values Using Cumulative Gaussian, Logistic, and Weibull Functions

\begin{tabular}{cccc}
\hline & \multicolumn{3}{c}{ Curve-Fitting Function } \\
\cline { 2 - 4 } Condition & Gaussian & Logistic & Weibull \\
\hline \multicolumn{4}{c}{ Participant H.W.U. } \\
Zero connected & $8.28153 \cdot 10^{-7}$ & $5.73142 \cdot 10^{-6}$ & $5.02623 \cdot 10^{-7}$ \\
One connected & $3.63179 \cdot 10^{-7}$ & $7.69745 \cdot 10^{-7}$ & $2.05849 \cdot 10^{-6}$
\end{tabular}

$3.63179 \cdot 10^{-7} \quad-7.69745 \cdot 10^{-7}-2.05849 \cdot 10^{-6}$ $\begin{array}{llll}\text { Two connected } & 0.01472 & 0.00422323 & 0.000879376\end{array}$

Participant A.M.Y.

Zero connected $\quad 3.89627 \cdot 10^{-7} \quad 1.53643 \cdot 10^{-6} \quad 0.000227812$ $\begin{array}{llll}\text { One connected } & 0.0112047 & 0.00356222 & 2.08027 \cdot 10^{-5}\end{array}$ $\begin{array}{llll}\text { Two connected } & 2.47041 \cdot 10^{-7} & 4.33683 \cdot 10^{-6} & 1.96182 \cdot 10^{-5}\end{array}$

Participant X.L.I.

Zero connected $\quad 0.000178414 \quad 8.6139 \cdot 10^{-7} \quad 0.000706466$ $\begin{array}{llll}\text { One connected } \quad 1.69017 \cdot 10^{-5} & 5.1101 \cdot 10^{-5} & 4.52698 \cdot 10^{-7}\end{array}$ $\begin{array}{lll}\text { Two connected } \quad 1.93216 \cdot 10^{-5} & 8.65 \cdot 10^{-6} & 8.87759 \cdot 10^{-6}\end{array}$

Participant Y.H.L.

Zero connected $\quad 6.63122 \cdot 10^{-6} \quad 2.68964 \cdot 10^{-6} \quad 1.51403 \cdot 10^{-6}$ $\begin{array}{llll}\text { One connected } & 2.45987 \cdot 10^{-7} & 2.71248 \cdot 10^{-6} & 2.00438 \cdot 10^{-5}\end{array}$ $\begin{array}{llll}\text { Two connected } & 0.00339481 & 0.000385675 & 1.05794 \cdot 10^{-7}\end{array}$

Stimuli. Stimuli were similar to those in Experiment 1, except that the total number of line segments in both test and reference patterns varied from four (in zero-connected patterns) to six (in twoconnected patterns), with the number of freely hanging lines held constant at four.

\section{Results}

After performing curve fitting for the psychometric functions using the cumulative Gaussian function, the PSEs for the zero-, one-, and two-connected conditions were $12.05,12.73$, and $12.99 ; 11.85,12.55$, and 12.74 ; and $11.96,12.42$, and 12.98 , respectively, for the 3 participants (see Figure 4). Note that the total number of lines present in each two-connected test pattern was now six, yet those patterns were still perceived as having lower numerosity (i.e., PSE $=12.99$ for Participant B.Z.Q.) than those in the zero- 
A

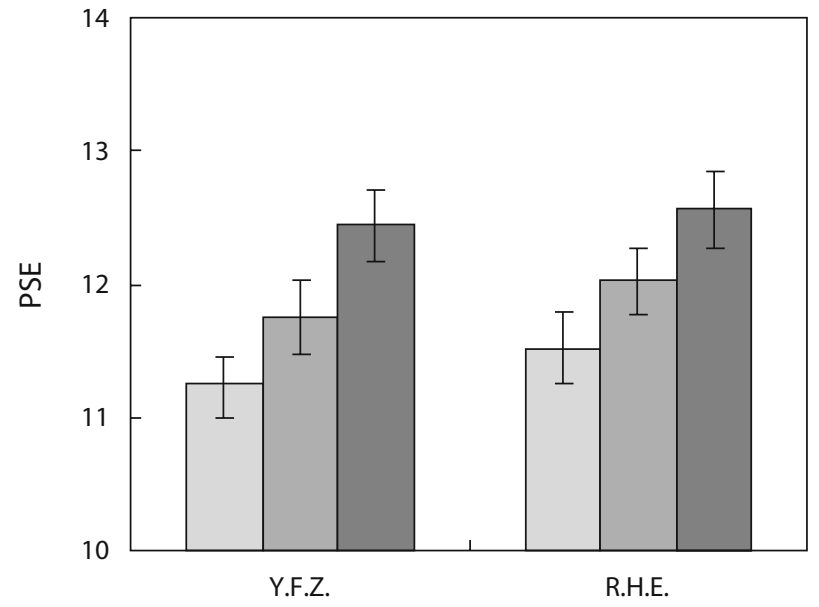

B

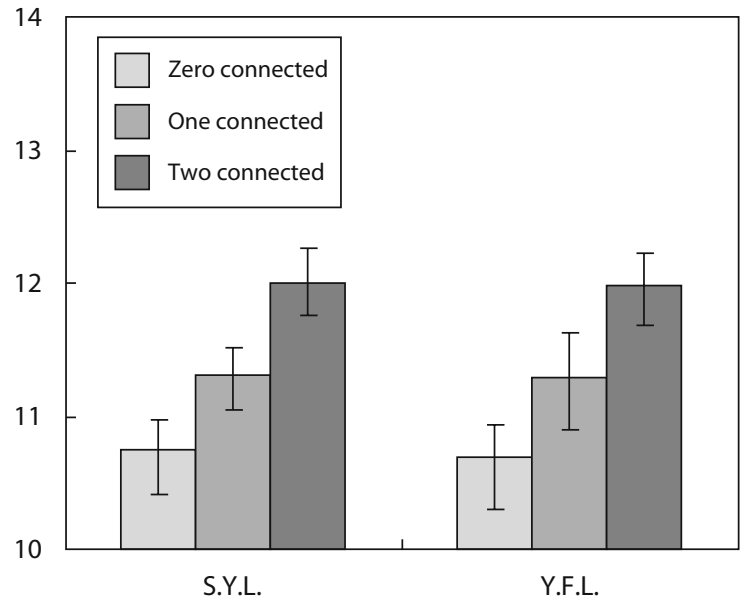

Figure 3. Point of subjective equality (PSE) values under different connected conditions for the 2 participants in Experiment $2 \mathrm{~A}$ (A) and for the 2 participants in Experiments 2B (B). Reference patterns were one- and two-connected patterns for Experiments $2 \mathrm{~A}$ and $2 B$, respectively, with a fixed number of dots (12).

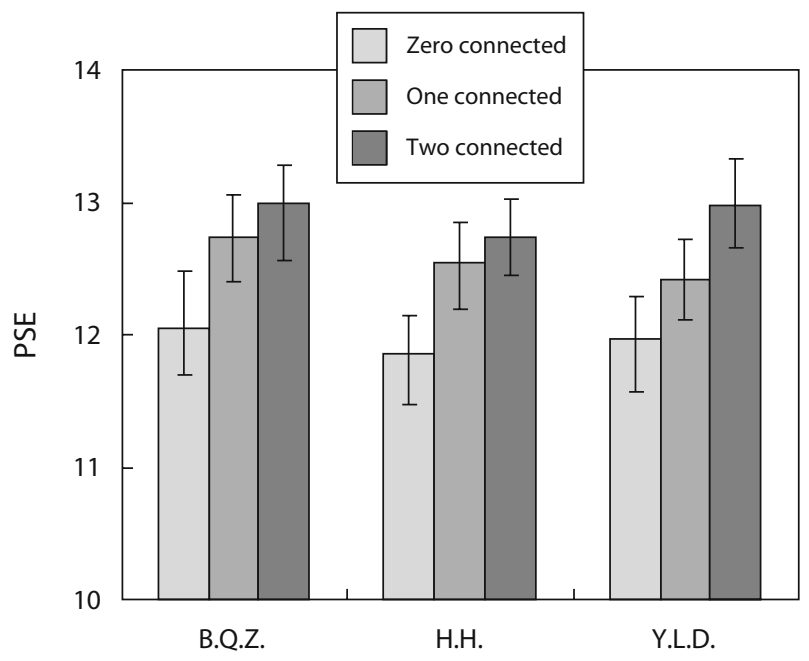

Figure 4. Point of subjective equality (PSE) values for Experiment 3 , in which the total number of freely hanging line segments was fixed at four for all test and reference patterns.

connected reference patterns with the same number of dots but with only four lines. Therefore, dot connectedness still affected numerosity judgments, although the effect was less pronounced, presumably because of the increased physical energy (number of lines) in the one- and two-connected test patterns compared with the zero-connected reference patterns.

\section{EXPERIMENT 4}

\section{Method}

Participants. Two university students participated in Experiment 4 , and they were both naive as to the purpose of the experiment. Neither had participated in any of the previous experiments.

Stimuli. Stimuli were similar to those of Experiment 1, except that test patterns did not contain pairs of connected dots. Instead, a line segment might have one end attached to a dot with the other end hanging freely to form a pin-like configuration (see Figure 5). Depending on the number of attaching dots and lines in a pattern, the test patterns were called zero pin, one pin, or two pins. Reference patterns were always zero pin with 12 dots.

\section{Results}

The three psychometric functions (based on the cumulative Gaussian function) are plotted in Figure 6. The PSEs for the zero-, one-, and two-pin conditions were 11.95, 12.16, and 12.19, respectively, for Participant Y.Q.G. and 11.96, 12.15, and 12.22, respectively, for Participant Z.Z. The three curves almost overlap with one another, although there are some slight differences for their PSEs, which suggests that the dots' merely being attached to lines cannot account for the underesti- 
A

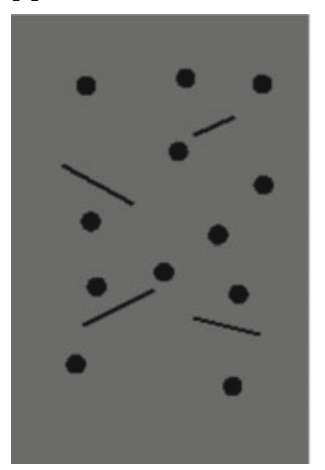

B

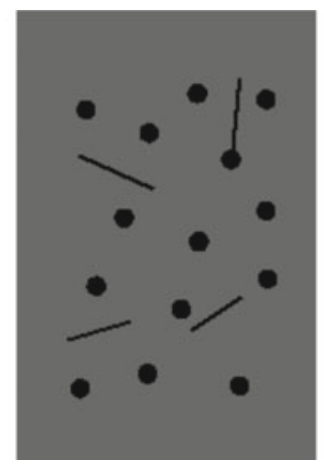

C

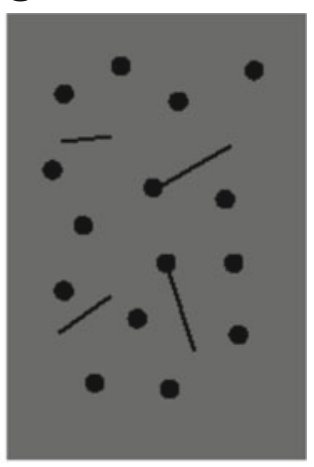

Figure 5. Examples of test patterns at different levels of dot attachment-zero pin (A), one pin (B), and two pins (C) - in Experiment 4.

A

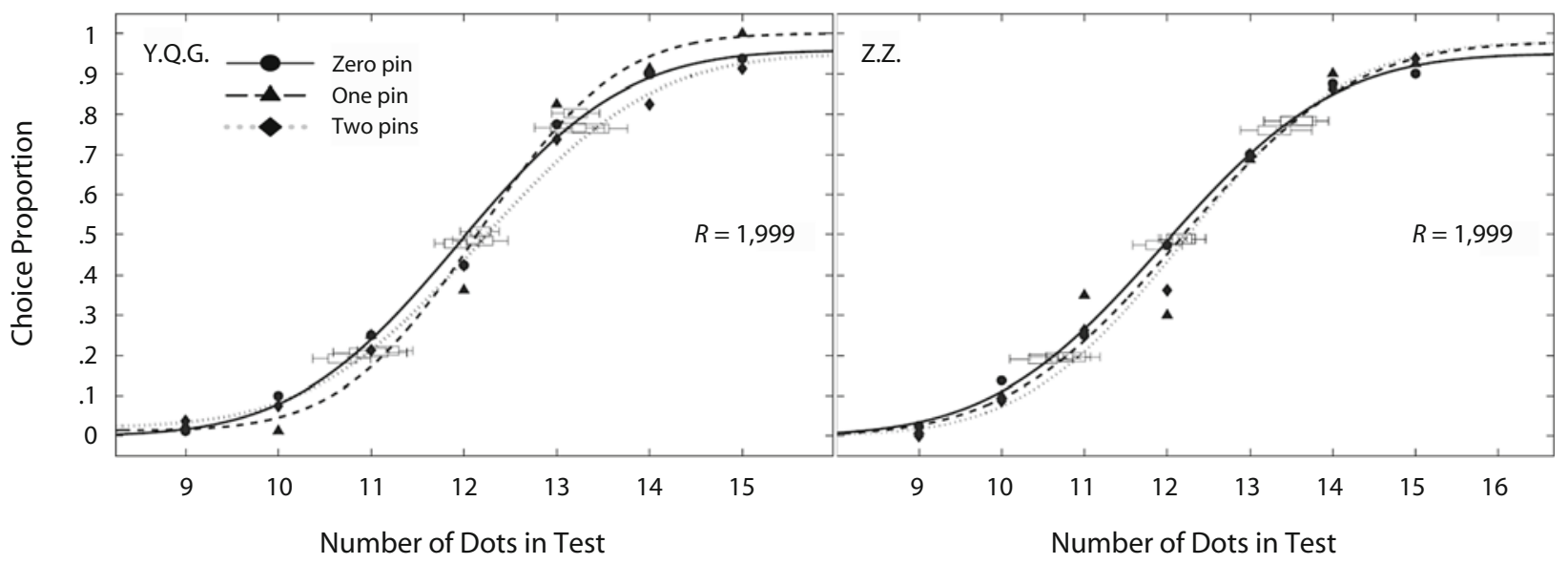

B

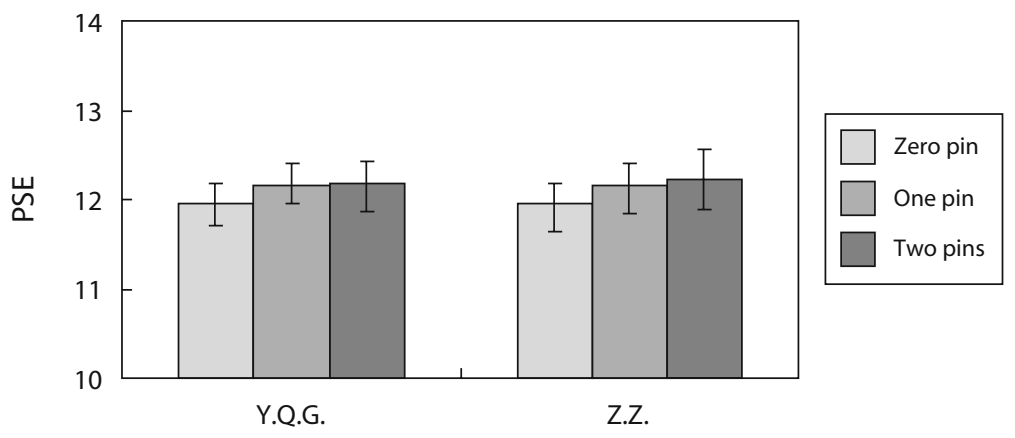

Figure 6. Psychometric functions (A) and point of subjective equality (PSE) values (B) in Experiment 4. There were no pairs of dots connected by lines; instead, one dot was attached to a line segment in the one-pin condition, and two dots were attached to a line segment in the two-pin condition. 


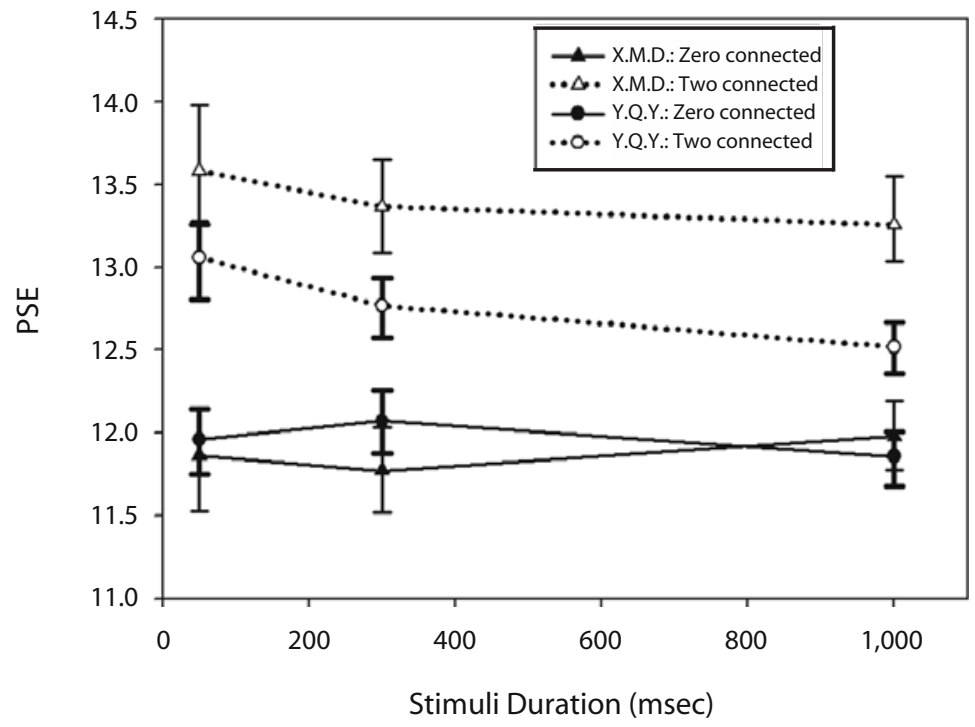

Figure 7. Point of subjective equality (PSE) values for zero- and two-connected conditions under different presentation durations $-50,300$, and $1,000 \mathrm{msec}-$ from Experiment 5.

mation of perceived numerosity reported in the previous experiments.

\section{EXPERIMENT 5}

\begin{abstract}
Method
Participants. Two graduate students participated in Experiment 5, and they were both naive as to the purpose of the experiment.

Stimuli. Stimuli were identical to those in Experiment 1, except that the presentation duration was manipulated in three conditions: 50,300 , and $1,000 \mathrm{msec}$. Because of the constraint on the total length of an experimental session, we included only visual patterns with zero or two pairs of connected dots (two levels of dot connectedness).
\end{abstract}

\section{Results}

The PSE values for the two conditions of dot connectedness are plotted against presentation time in Figure 7. Underestimation occurred in three duration conditions: PSE $=1.72,1.59$, and 1.28 for Participant X.M.D.; and PSE $=1.10,0.70$, and 0.66 for Participant Y.Q.Y. Despite the slight trend of a decrease with increasing stimulus duration, connectedness consistently affected dot numerosity judgments across a 20 -fold difference from 50 to $1,000 \mathrm{msec}$.

\section{DISCUSSION}

In this study, we investigated the effect of the connectivity of dots on numerosity judgments for a visual display using a two-alternative forced choice paradigm. Results show that connecting pairs of dots leads to an underestimation of the total number of dots, with the effect size directly related to the number of pairs of connected dots. Underestimation of dot numerosity occurred when the dots in the reference patterns were themselves either connected (Experiment 2) or not connected (Experiment 1) and when the number of freely hanging lines was controlled for (Experiments 2A and 2B). Underestimation was not remarkable when the dots were merely attached to one end of a line segment (Experiment 4). It did occur quite consistently, however, across a significant span of presentation durations, from 50 to $1,000 \mathrm{msec}$ (Experiment 5). Taken together, this series of experiments have conclusively established that it is the dot connection per se that mediates the underestimation effect.

Existing mechanisms for numerosity perception cannot adequately account for our results. First, exact enumeration based on distinct items, including one-by-one verbal counting and subitization (a process of immediate and accurate recognition of small numbers), was not used in the present situation because of the brief stimulus presentation duration and the moderate amount of dots (9-15) in the display. It is known that counting requires a serial shift of spatial attention and is effortful and error prone (Trick \& Pylyshyn, 1994), whereas numerosity perception relies on parallel processes, which is revealed by how set size influences reaction times in humans (Barth, Kanwisher, \& Spelke, 2003) and eye movements (Nieder \& Miller, 2004) and neural response latencies (Nieder, Freedman, \& Miller, 2004) in monkeys. It is possible that counting, which requires attention to be deployed to items sequentially, is facilitated by perceptual grouping of dots through connecting them, because perceptual groups direct attention. However, the possibility that attention is driven by connectedness cannot account for the pattern of numerosity underestimation found here, which is indirectly supported by the results from our manipulation of presentation duration (Experiment 5). On the other hand, the occupancy model (Allik \& Tuulmets, 1991), which attributes perceived numerosity to the amount of spatial coverage by dots (and, for our stimuli, possibly to line segments that also might contribute an extensive amount of spatial coverage), cannot explain why increasing the number of freely hanging line segments does not reverse 
the underestimation effect (Experiment 3), whereas attaching dots to line segments abolishes the same amount of underestimation effect (Experiment 4). It seems that there might be two different stages underlying numerosity perception: first, the individuation of items in a visual display, followed by magnitude estimation based on the distinct number of items (see, e.g., Gallistel \& Gelman, 2000). According to this view, numerosity judgments depend on the number of distinct objects being perceived, and the underestimation effect reported here may reflect a perceptual organization process through which distinct objects are formed.

Palmer and Rock (1994) proposed the principle of uniform connectedness for perceptual organization; that is, a connected region of homogeneous visual properties corresponds to the entry-level units of visual stimuli. Chen $(1982,1985,2005)$ advanced the topological theory of visual perception, which posits a pivotal role for connectivity (as a topological property) in object perception. Our experimental findings support these theoretical positions: During brief presentation, a pair of connected dots tends to be processed as an integrated whole rather than as two individual dots joined by a line. Such holistic, configural processing (e.g., Kubovy \& Gepshtein, 2003; Pomerantz, 2006; Wenger \& Townsend, 2001), we argue, leads to underestimation of the number of objects (dots) in the display. Future experiments will illuminate the exact temporal conditions under which the underestimation effect occurs as a consequence of the binding of dots by line segments.

\section{AUTHOR NOTE}

This project was supported by Ministry of Science and Technology of China Grants 2005CB522800 and 2004CB318101, National Nature Science Foundation of China Grant 30621004, and Knowledge Innovation Projects Grant CSTD S2002-1 from the Chinese Academy of Sciences, with L.C. as the principal investigator and J.Z. as an overseas guest investigator. Correspondence concerning this article should be addressed to J. Zhang, Department of Psychology, University of Michigan, Ann Arbor, MI 48109 (e-mail: junz@umich.edu), or to the first author (lxhe@cogsci.ibp.ac.cn).

\section{REFERENCES}

Allik, J., \& Tuulmets, T. (1991). Occupancy model of perceived numerosity. Perception \& Psychophysics, 49, 303-314.

Allik, J., Tuulmets, T., \& Vos, P. G. (1991). Size invariance in visual number discrimination. Psychological Research, 53, 290-295.

Barth, H., Kanwisher, N., \& Spelke, E. (2003). The construction of large number representations in adults. Cognition, 86, 201-221.

BuRgESS, A., \& BARLOW, H. B. (1983). The precision of numerosity discrimination in arrays of random dots. Vision Research, 23, 811-820.

Chen, L. (1982). Topological structure in visual perception. Science, 218, 699-700.

CHEN, L. (1985). Topological structure in the perception of apparent motion. Perception, 14, 197-208.
CHEN, L. (2005). The topological approach to perceptual organization. Visual Cognition, 12, 553-637.

Gallistel, C. R., \& Gelman, R. (2000). Non-verbal numerical cognition: From reals to integers. Trends in Cognitive Sciences, 4, 59-65.

GinsBurg, N. (1978). Perceived numerosity, item arrangement, and expectancy. American Journal of Psychology, 91, 267-273.

Ginsburg, N., \& Deluco, T. (1979). A developmental study of the regular-random numerosity illusion. Journal of Genetic Psychology, 135, 197-201.

GinsBURG, N., \& GoldDSTEIN, S. R. (1987). Measurement of visual cluster. American Journal of Psychology, 100, 193-203.

Ginsburg, N., \& Nicholls, A. (1988). Perceived numerosity as a function of item size. Perceptual \& Motor Skills, 67, 656-658.

INDOw, T., \& IDA, M. (1977). Scaling of dot numerosity. Perception \& Psychophysics, 22, 265-276.

Koesling, H., Carbone, E., Pomplun, M., Sichelschmidt, L., \& RitTER, H. (2004). When more seems less - non-spatial clustering in numerosity estimation. In Proceedings of the Early Cognitive Vision Workshop, May 28-June 1, 2004. Isle of Skye, Scotland.

Koesling, H., Pomplun, M., \& Ritter, H. (1998). The effects of structural information on perceived numerosity in two-dimensional object distributions (CRC 360 Technical Report 2/1998). Bielefeld, Germany: University of Bielefeld.

Krueger, L. E. (1972). Perceived numerosity. Perception \& Psychophysics, 11, 5-9.

Krueger, L. E. (1984). Perceived numerosity: A comparison of magnitude production, magnitude estimation, and discrimination judgments. Perception \& Psychophysics, 35, 536-542.

Kubovy, M., \& Gepshtein, S. (2003). Perceptual grouping in space and in space-time: An exercise in phenomenological psychophysics. In R. Kimchi, M. Behrmann, \& C. R. Olson (Eds.), Perceptual organization in vision: Behavioral and neural perspectives (pp. 45-86). Mahwah, NJ: Erlbaum.

Nieder, A., Freedman, D. J., \& Miller, E. K. (2004). Representation of the quantity of visual items in the primate prefrontal cortex. Science, 297, 1708-1711.

Nieder, A., \& Miller, E. K. (2004). Analog numerical representations in Rhesus monkeys: Evidence for parallel processing. Journal of Cognitive Neuroscience, 16, 889-901.

PAlmer, S., \& Rock, I. (1994). Rethinking perceptual organization: The role of uniform connectedness. Psychonomic Bulletin \& Review, 1, 29-55.

Pomerantz, J. R. (2006, November). Studying configural processing and gestalts: What are the challenges? Paper presented at the first meeting of the Configural Processing Consortium, Houston, TX.

TRICK, L. M., \& Pylyshyn, Z. W. (1994). Why are small and large numbers enumerated differently? A limited-capacity preattentive stage in vision. Psychological Review, 101, 80-102.

Wenger, M. J., \& Townsend, J. T. (2001). Faces as gestalt stimuli: Process characteristics. In M. J. Wenger \& J. T. Townsend (Eds.), Computational, geometric, and process perspectives on facial cognition: Contexts and challenges (pp. 229-284). Mahwah, NJ: Erlbaum.

Wichmann, F. A., \& Hill, N. J. (2001a). The psychometric function: I. Fitting, sampling, and goodness of fit. Perception \& Psychophysics, 63, 1293-1313.

Wichmann, F. A., \& Hill, N. J. (2001b). The psychometric function: II. Bootstrap-based confidence intervals and sampling. Perception \& Psychophysics, 63, 1314-1329.

(Manuscript received November 10, 2007; revision accepted for publication December 3, 2008.) 